\title{
Cyclin A as a marker for prognosis and chemotherapy response in advanced breast cancer
}

\author{
P Poikonen ${ }^{*, 1}$, J Sjöström', R-M Amini ${ }^{2}, K_{\text {Villman }}{ }^{3}$, J Ahlgren $^{4}$ and C Blomqvist' \\ 'Department of Oncology, Helsinki University Central Hospital, PO Box 180, 00029 HUS Helsinki, Finland; '2Department of Genetics and Pathology, \\ Uppsala University Hospital, Uppsala, Sweden; ${ }^{3}$ Department of Oncology, Medical Centre Hospital, Örebro, Sweden; ${ }^{4}$ Department of Oncology, Gävle \\ Hospital and Clinical Research Centre, County of Gävleborg, Gävleborg, Sweden
}

\begin{abstract}
We wanted to study cyclin A as a marker for prognosis and chemotherapy response. A total of 283 women with metastatic breast cancer were initially enrolled in a randomised multicentre trial comparing docetaxel to sequential methotrexate-fluorouracil (MF) in advanced breast cancer after anthracycline failure. Paraffin-embedded blocks of the primary tumour were available for 96 patients (34\%). The proportion of cells expressing cyclin A was determined by immunohistochemistry using a mouse monoclonal antibody to human cyclin A. Response evaluation was performed according to WHO recommendations. The median cyclin A positivity of tumour cells was 14.5\% (range 1.2-45.0). Cyclin A correlated statistically significantly to all other tested proliferation markers (mitotic count, histological grade and Ki-67). A high cyclin A correlated significantly to a shorter time to first relapse, risk ratio (RR) I.94 (95\% Cl I.24-3.03) and survival from diagnosis, RR 2.49 (95\% Cl I.45-4.29), cutoff point for high/low proliferation group I0.5\%. Cyclin A did not correlate to chemotherapy response or survival after anthracycline, docetaxel or MF therapy. Of all tumour biological factors tested (mitotic count, histological grade and Ki-67), cyclin A seemed to have the strongest prognostic value. Cyclin A is a good marker for tumour proliferation and prognosis in breast cancer. In the present study, cyclin A did not predict chemotherapy response. British Journal of Cancer (2005) 93, 515-519. doi:I0.1038/sj.bjc.6602735 www.bjcancer.com

Published online 9 August 2005

(c) 2005 Cancer Research UK
\end{abstract}

Keywords: cyclin A; advanced breast cancer; prognosis; chemotherapy response; proliferation

Chemotherapy has an essential role in the treatment of both local and advanced breast cancer (Anonymous, 1998; Crown et al, 2002). Since not all patients benefit from aggressive chemotherapy, identifying factors predicting poor prognosis and chemotherapy response would be of most importance. The measurements of the proliferative cell fraction are widely used to evaluate the tumour growth rate and to establish the prognosis in cancer patients. High proliferation activity correlates strongly with poor prognosis in breast cancer, irrespective of the methodology used. According to a recent review, counting the cells in mitosis is the most reproducible and independent of the various markers for tumour proliferation, while Ki-67 labelling and cyclin A index are promising alternatives (van Diest et al, 2004). Mitotic count is the most important constituent of the histological grade (Genestie et al, 1998).

Cyclins and cyclin-dependent kinases regulate the cell cycle at specific points. Cyclin $\mathrm{A}$ is essential in the $\mathrm{S}$ phase for DNA replication, it is also involved in G2-M-phase transition (Zindy et al, 1992; Fan and Bertino, 1997), and is therefore one of the most useful markers signifying proliferating cells. Overexpression of cyclin A has been associated with worse prognosis for breast cancer in several studies (Bukholm et al, 2001; Michalides et al, 2002; Michels et al, 2002), but the results have not been consistent (Kuhling et al, 2003; Rudolph et al, 2003). Since the lack of prognostic significance of cyclin A is mainly seen in patients with

*Correspondence: Dr P Poikonen; E-mail: Paula.Poikonen@hus.fi Revised 31 May 2005; accepted 6 July 2005; published online 9 August 2005 lymph node-negative breast cancer, it is possible that cyclin A plays a more important role in aggressive tumours and metastatic disease (Kuhling et al, 2003).

Of various tumour biological factors, including different proliferation markers, only the S-phase fraction seems to be uniformly associated with response to preoperative chemotherapy, but not in metastatic disease (Sjöström, 2002). No predictive markers for the chemotherapy response are presently in use in the treatment of advanced breast cancer.

High cyclin A score predicted a better chemotherapy response and longer progression-free survival in patients with soft tissue sarcoma (Huuhtanen et al, 1999). To our knowledge, there are no studies of cyclin A as a predictive marker for chemotherapy response in metastatic breast cancer. The purpose of the present study is to evaluate cyclin A as a marker for tumour aggressiveness and chemotherapy response in patients with advanced breast cancer treated in a randomised trial (Sjöström et al, 1999).

\section{PATIENTS AND METHODS}

\section{Patients}

The study is based on a phase III multicentre trial, where 140 patients were randomly allocated to receive intravenous methotrexate and 5-fluorouracil, and 143 to receive intravenous docetaxel until disease progression as second-line therapy for metastatic breast cancer between December 1994 and October 1997 (Sjöström et al, 1999). The patients were required to have 
histologically proven primary breast cancer that had progressed during or after the first-line anthracycline-containing treatment for metastatic disease, or that had relapsed within 12 months after discontinuation of adjuvant anthracycline-containing regimen. The patients had to be $18-70$ years old with a performance status $\leqslant 2$, and have no more than one previous chemotherapy regimen for advanced disease (multiple endocrine treatments and radiotherapy were allowed). Patients with measurable lesions or evaluable lesions were eligible. Response evaluation for docetaxel and methotrexate-fluorouracil (MF) treatment was carried out during every third course, at treatment discontinuation and every 3 months during follow-up. Anthracycline response was evaluated retrospectively from patient documents. Response evaluation was performed according to WHO recommendations (Miller et al, 1981). All patients with histological blocks from the primary tumour available for analysis were included in this study. All stainings were reviewed, classified and regraded by one of the authors (RMA), who did not have access to clinical data. Characteristics of the primary tumours at the time of diagnosis are shown in Table 1. Mitotic grading into three classes (1-3) was carried out according to the recommendations of Elston and Ellis (1991). Ethical approval was obtained for the randomised chemotherapy study, including subsequent studies on biological markers.

\section{Immunohistochemical assays}

Paraffin-embedded blocks of the primary tumour were available for 96 patients (34\%). All tissues had been fixed in $4 \%$ buffered formalin, processed and embedded in paraffin. From each block, $5-\mu \mathrm{m}$-thin sections were cut on coated slides and dried overnight at $37^{\circ} \mathrm{C}$. Sample deparaffination was performed in xylene and rehydration in alcohol to distilled water. Antigen demasking was carried out by heating the samples in a microwave oven $(850 \mathrm{~W})$ in citric acid buffer ( $\mathrm{pH}$ 6.0) for $20 \mathrm{~min}$, buffer was added in need.

Table I Characteristics of the primary tumours at the time of diagnosis and time to first relapse (TFR) of the 96 investigated patients

\begin{tabular}{lcc}
\hline Factor & No. of patients (\%) & Median (range) \\
\hline Histology & \\
Ductal & $94(98)$ \\
Lobular & $2(2)$ \\
Oestrogen receptor status & \\
Positive & $48(50)$ \\
Negative & $39(41)$ \\
Unknown & $9(9)$ \\
Mitotic count & \\
I & $12(13)$ \\
2 & $29(30)$ \\
3 & $55(57)$ \\
Tumour grade & \\
1 & $1(1)$ \\
2 & $32(33)$ \\
3 & $63(66)$ \\
KI-67 & \\
$<25 \%$ & $29(30)$ \\
$\geqslant 25 \%$ & $67(70)$ \\
Cyclin A & \\
$<10.5 \%$ & $33(34)$ \\
$\geqslant 10.5 \%$ & $63(66)$ \\
TFR (years) & & \\
\hline
\end{tabular}

Treatment with $1.6 \%$ methanolperoxidase was used to inhibit endogenous peroxidase activity. For immunohistochemistry (IHC), the specimens were incubated overnight at room temperature with 1:100 diluted mouse monoclonal antibody to human cyclin A (Novocastra, Newcastle, UK) and for Ki-67 determination with 1:500 mouse anti-human monoclonal Mib-1 antibody (Immunotech, Marseille, France). The binding of the primary antibody was detected by a peroxidase-conjugated secondary antibody using an Elite ABC Kit (Vectastain, Vector Laboratories, Burlingame, CA, USA). As positive and negative controls for cyclin A stainings, we used hyperplastic tonsil tissue in cyclin A stainings. The primary antibody was omitted from negative controls.

For quantification of the immunostaining, the tumour area with the highest density of positive nuclear staining was chosen. To calculate the percentage of positively stained nuclei, an ocular grid of $100(10 \times 10)$ squares was used at $10 \times 40$ magnification. All positive nuclei from this area were counted. To estimate the negative nuclei in the same area of 100 squares, three different rows of 10 squares were counted and the mean score multiplied by 10. In the case of tumours with scarce cellularity, several fields were evaluated, and negative nuclei were counted from the whole grid area of 100 squares. The percentage of positive nuclei was estimated by dividing the number of positively stained cells by the entire number of cells in the same area.

\section{Statistical methods}

Statistical analyses were carried out using Macintosh SPSS statistical software package. The association of TNM stage and tumour characteristics with cyclin A was tested with Spearman correlation coefficient for ordinal variables and Student's $t$-test for dichotomous variables. The association between different proliferation markers was tested by computing the nonparametric Spearman correlation coefficient. The association between treatment response and proliferation markers was measured by computing the Pearson correlation coefficient with CR classified as $4, \mathrm{PR}$ as $3, \mathrm{NC}$ as 2 and $\mathrm{PD}$ as 1 , and proliferation markers as continuous variables. Overall survival (OS) and time to first relapse (TFR) curves were prepared by the Kaplan-Meier method, and prognostic variables were tested using the Cox regression analysis with proliferation markers as continuous variables. Due to multiple comparisons, a significance level of 0.01 was chosen. The impact of proliferation rate and grade on TTP and OS was tested with rates as both continuous and discrete (high/low) variables. The results were similar with these two methods. Groups with high and low proliferation rates were divided with cutoff points corresponding to Ki-67 of $25 \%$, with the aid of linear regression analysis. A linear equation of the form $y=k x$ was formed by regressing cyclin A and mitotic grade, respectively, against Ki-67. The cutoff point was obtained by inserting the value of 25 as $x$ and solving the equation for $y$. Consequently, the cutoffs for the three different proliferation measurements were $25,10.5 \%$ and grade 3 for $\mathrm{Ki}-67$, cyclin A and mitotic grade, respectively. These cutpoints identified about $65 \%$ in the high-risk groups for all markers (Table 1).

\section{RESULTS}

The median cyclin A positivity of tumour cells was $14.5 \%$ (range $1.2-45.0)$. The frequency distributions of all tested proliferation markers (mitotic count, histological grade, Ki-67 and cyclin A) are presented in Table 1. Tumour characteristics at the time of diagnosis according to cyclin A positivity are presented in Table 2. All proliferation markers correlated highly statistically significantly to each other, $P<0001$. The strongest correlation was between Ki-67 and cyclin A, Spearman correlation coefficient 0.74 . 
Table 2 Tumour characteristics at the time of diagnosis according to cyclin A positivity

\begin{tabular}{|c|c|c|c|}
\hline Tumour characteristic & $\begin{array}{c}\text { Cyclin } \\
\text { A }<10.5 \% \\
\text { No. of } \\
\text { patients (\%) }\end{array}$ & $\begin{array}{c}\text { Cyclin } \\
\text { A }>10.5 \% \\
\text { No. of } \\
\text { patients (\%) }\end{array}$ & $P$-value \\
\hline \multicolumn{4}{|l|}{ Tumour size } \\
\hline $0-2 \mathrm{~cm}$ & $10(30)$ & $14(22)$ & \\
\hline $2.1-5 \mathrm{~cm}$ & $12(36)$ & $29(46)$ & \\
\hline$>5 \mathrm{~cm}$ & $4(12)$ & $9(14)$ & \\
\hline $\begin{array}{l}\text { Direct extension to skin } \\
\text { or chest wall }\end{array}$ & $2(6)$ & $4(6)$ & NS \\
\hline Unknown & $5(15)$ & $7(11)$ & \\
\hline \multicolumn{4}{|l|}{ Nodal status } \\
\hline Negative & $12(36)$ & $19(30)$ & \\
\hline Positive & $18(55)$ & $36(57)$ & \\
\hline Fixed lymph nodes & $2(6)$ & $7(11)$ & \\
\hline Unknown & I (3) & I (2) & NS \\
\hline \multicolumn{4}{|l|}{ Primary metastatic disease } \\
\hline No & $23(70)$ & $5 \mid(8 I)$ & \\
\hline Yes & $6(18)$ & $7(11)$ & \\
\hline Unknown & $4(12)$ & $5(8)$ & NS \\
\hline \multicolumn{4}{|l|}{ Hormone receptor status } \\
\hline ER+ & $10(30)$ & $29(46)$ & \\
\hline ER- & $21(64)$ & $27(43)$ & \\
\hline Unknown & $2(6)$ & $7(11)$ & $P=0.003$ \\
\hline \multicolumn{4}{|l|}{ Histology } \\
\hline Ductal & $31(94)$ & $63(100)$ & \\
\hline Lobular & $2(6)$ & & \\
\hline \multicolumn{4}{|l|}{ c-erb-B2 } \\
\hline Low & $5(15)$ & $29(46)$ & \\
\hline Intermediate & $3(9)$ & $16(25)$ & \\
\hline High & $11(33)$ & $18(28)$ & NS \\
\hline \multicolumn{4}{|l|}{ Grade } \\
\hline 1 & $3(9)$ & | (2) & \\
\hline 2 & $17(52)$ & $13(21)$ & \\
\hline 3 & $13(39)$ & $49(78)$ & $P<0.001$ \\
\hline
\end{tabular}

Table 3 Spearman correlation coefficient and significance between the investigated tumour biological factor

\begin{tabular}{lccc}
\hline & Grade & KI-67 & Cyclin A \\
\hline Mitotic count & 0.82 & 0.39 & 0.39 \\
Grade & & 0.36 & 0.40 \\
KI-67 & & & 0.74 \\
\hline
\end{tabular}

P-value 0.001 for all comparisons.

Spearman correlation coefficients between tumour proliferation markers are presented in Table 3.

Out of 96 patients, 58 were evaluable for response after first-line anthracycline treatment and 96 after second-line docetaxel or MF treatment (50 in the docetaxel group and 46 in the MF group). The overall response rate $(\mathrm{CR}+\mathrm{PR})$ was $47 \%$ after first-line anthracycline therapy, $46 \%$ in the docetaxel arm and $26 \%$ in the MF arm, respectively. In the parent study $(n=283)$, the corresponding response rates for docetaxel and MF treatment were 42 and $21 \%$ (Sjöström et al, 1999). Association of the overall response rate (complete or partial response) with the investigated tumour

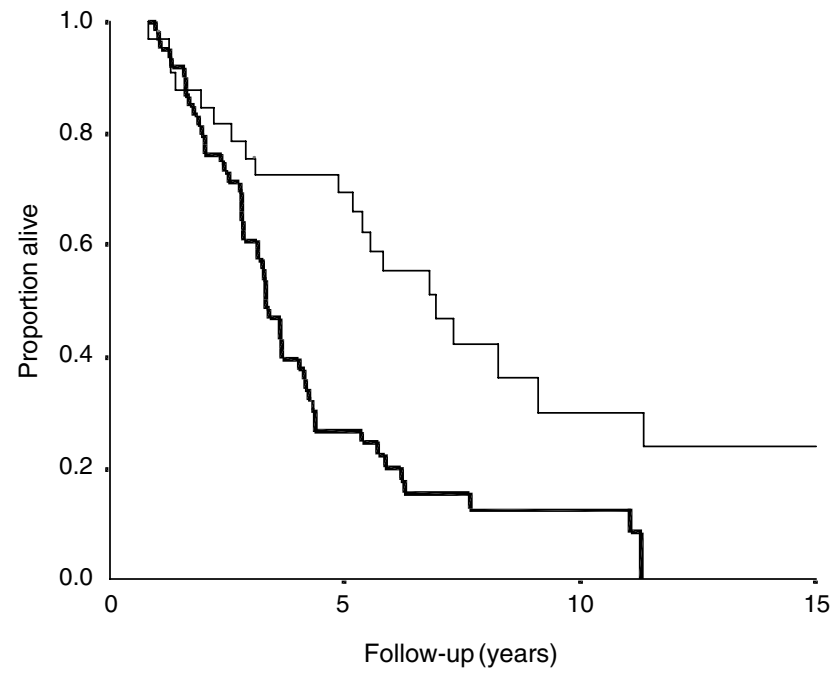

Figure I Kaplan-Meier plot for OS from diagnosis according to cyclin A. Thin line, cyclin $A<10.5 \%$; thick line, cyclin $A>10.5 \%, P=0.004$.

proliferation markers is shown in Table 4. There was no significant correlation between chemotherapy response and any proliferation marker after anthracycline, docetaxel or MF treatment.

There was a significant association between a high cyclin A score and TFR, risk ratio (RR) $1.03(95 \%$ CI $1.01-1.05), P=0.001$ (as continuous variable) and RR 1.94 (95\% CI 1.24-3.03), $P=0.004$ (as discrete variable), while the other markers showed only nonsignificant trends in the same direction. There was also a highly significant association between a high Cyclin A score and OS from diagnosis (Figure 1), RR 1.05 (95\% CI 1.02-1.07), $P<0.001$ (as continuous variable) and RR 2.49 (95\% CI 1.45-4.29), $P=0.004$ (as discrete variable), but not for survival from start of first- or second-line chemotherapy. For details, see the results presented in Table 5. Since the prognostic value of cyclin A seemed to depend on chemotherapy, we separately analysed the association between cyclin $\mathrm{A}$ and whether the patient had received adjuvant chemotherapy or not. While the prognostic impact of cyclin A on survival from diagnosis was significant for both groups, the RR was somewhat higher in patients who did not receive adjuvant chemotherapy RR 1.07 (95\% CI $1.03-1.10)$ compared to patients who were given adjuvant chemotherapy RR 1.03 (95\% CI $1.00-1.06)$.

\section{DISCUSSION}

The main finding of the present study was the significant association of a high cyclin A with a short TFR and poor survival. The difference in survival from diagnosis of breast cancer was quite remarkable, despite the fact that all patients developed metastatic disease, since the patient material was recruited from a study in metastatic disease. We used a cutoff value of $10.5 \%$ for the high and low cyclin A positivity groups. This is in line with three other studies, where median values varying between 8 and $11 \%$ have been used (Michalides et al, 2002; Michels et al, 2002; Kuhling et al, 2003; Rudolph et al, 2003). In the fourth study, four different categories were used for cyclin A positivity and the median category was between 15 and 30\% (Bukholm et al, 2001).

We found a strong correlation between cyclin A, mitotic count, tumour grade and Ki-67, which is in line with previous studies in breast cancer (Michalides et al, 2002; Michels et al, 2002). Cyclin A was the only marker that showed a statistically significant correlation to both TFR and OS; therefore, it seems to be the most useful marker of proliferation. There are some well-known 
Table 4 Association of the overall response rate (complete or partial response) to different chemotherapy with the investigated tumour biological factors in patients with evaluable response to treatment

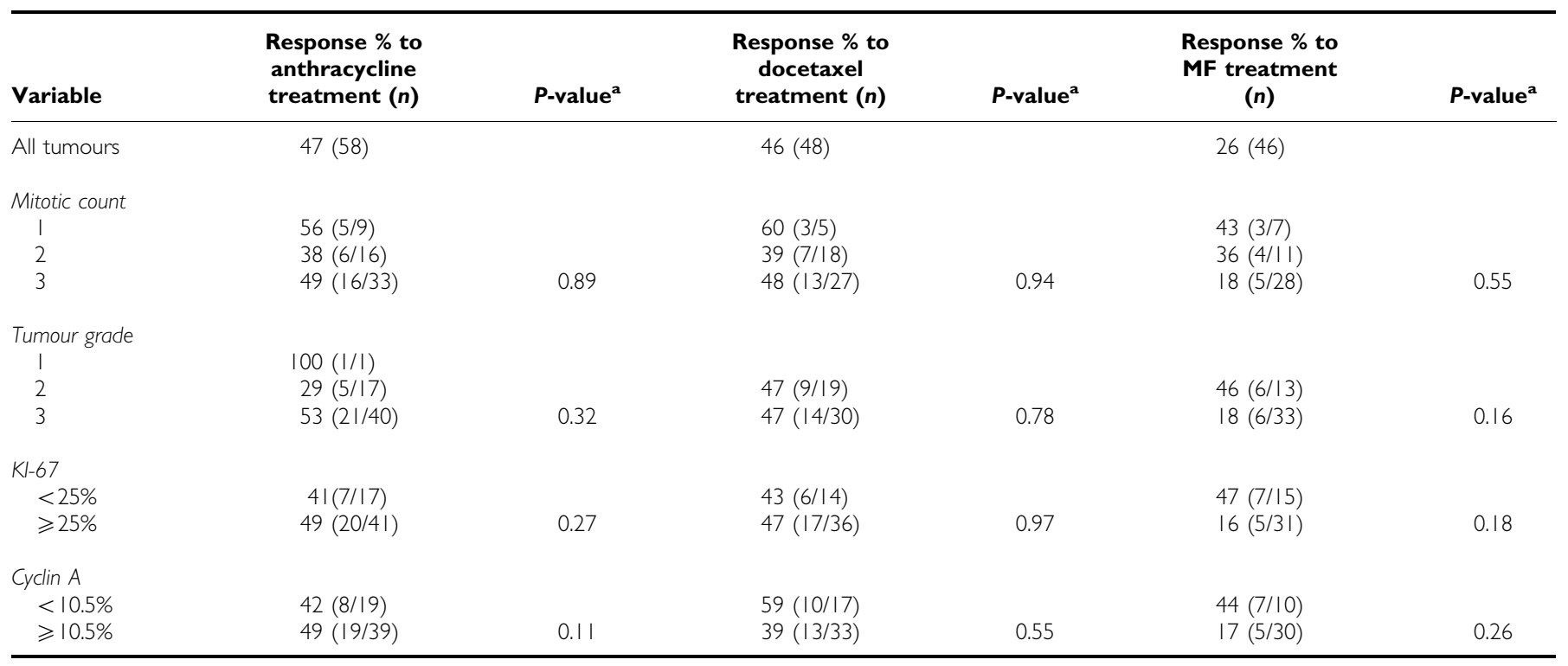

${ }^{\mathrm{a}} \mathrm{P}$-value for the Pearson correlation coefficient.

Table 5 Cox regression analysis according to tumour biological factor (as discrete, high/low variable) and different chemotherapy regimen

\begin{tabular}{|c|c|c|c|c|}
\hline \multicolumn{5}{|c|}{ Risk ratio $(95 \% \mathrm{Cl})$} \\
\hline & Grade & Mitotic count & KI-67 & Cyclin A \\
\hline OSdg & $1.95(1.16-3.27)$ & $1.62(0.99-2.65)$ & $1.61(0.95-2.70)$ & $2.49(1.45-4.29)$ \\
\hline \multicolumn{5}{|c|}{ Efficacy parameters after anthracycline treatment } \\
\hline TTPa & $0.46(0.22-0.97)$ & $0.82(0.42-1.60)$ & $0.83(0.40-1.71)$ & $0.63(0.31-1.27)$ \\
\hline \multicolumn{5}{|c|}{ Efficacy parameters after docetaxel or MF treatment } \\
\hline TTPdoc & $1.02(0.56-1.87)$ & $0.83(0.46-1.51)$ & $0.63(0.32-1.24)$ & $1.32(0.70-2.48)$ \\
\hline OSdoc & $1.46(0.75-2.86)$ & $0.65(0.61-2.21)$ & $0.85(0.41-1.76)$ & $1.90(0.90-4.06)$ \\
\hline TTPmf & $1.30(0.66-2.54)$ & $1.08(0.58-2.00)$ & $1.19(0.62-2.26)$ & $1.20(0.64-2.26)$ \\
\hline OSmf & $1.25(0.56-2.83)$ & $0.92(0.46-2.02)$ & $1.14(0.53-2.46)$ & $1.01(0.48-2.13)$ \\
\hline
\end{tabular}

TFR: time to first relapse, TTPa: time to progression after anthracycline therapy, OSa: overall survival after anthracycline therapy, TTPdoc: time to progression after docetaxel therapy, OSdg: overall survival from diagnosis, OSdoc: overall survival after docetaxel therapy, MF: methotrexate fluorouracil, TTPmf: time to progression after methotrexate fluorouracil therapy, OSmf: overall survival after methotrexate fluorouracil therapy.

problems with the use of traditional markers for proliferation in breast cancer. The duration of mitotic phase can vary and the mitotic count is not linearly correlated to proliferating cells, especially in aneuplodic tumours. Although histological grade is a well-established prognostic factor, the reproducibility of the method has been questioned (Boiesen et al, 2000). The flow-cytometric determination of $S$ phase requires tumour volumes larger than immunohistochemical methods, and there is also pronounced intratumoral heterogenity in $S$ phase (van Diest et al, 2004). Moreover, fresh frozen tissue is needed for S-phase analysis and the costs for flow cytometry are higher than for IHC. Cyclin A is expressed during the late S, G2 and M phases, and it is therefore a useful marker for proliferating cells (Zindy et al, 1992; Fan and Bertino, 1997). Cyclin A can be analysed on paraffin-embedded tissue with the immunohistochemial technique, which is an advantage both for practical and economical reasons.

An association between a high cyclin A expression and poor prognosis has previously been described in three studies, all executed after primary therapy for breast cancer (Bukholm et al, 2001; Michalides et al, 2002; Michels et al, 2002). A fourth study, however, in node-negative patients showed no correlation between cyclin A and prognosis (Kuhling et al, 2003; Rudolph et al, 2003). Several studies have revealed an association between a high proliferation index measured by other means and favourable chemotherapy response. In the preoperative setting, most studies have shown that a higher proliferation index is associated to a higher response rate of anthracycline-based chemotherapy, while the results in metastatic breast cancer are less consistent (Sjöström, 2002). There are data also from other malignancies, indicating that a higher proliferation rate correlates to a better chemotherapy response (Joensuu et al, 1994; Hahka-Kemppinen et al, 1997; Huuhtanen et al, 1999). We are aware of no clinical data on cyclin $\mathrm{A}$ as a predictive factor for chemotherapy in breast cancer. A preclinical study by Volm showed that breast cancer cell lines with high cyclin A were significantly more sensitive to doxorubicin than cell lines with low cyclin A activity (Volm et al, 1997). 
Anthracyclines are active throughout the cell cycle, but the effects are most pronounced for cells in S or G2 phase. The antimetabolites methotrexate and 5-fluorouracil as antimetabolites mainly inhibit the cell proliferation in the $S$ phase, and docetaxel, which is a microtubulin stabiliser, exerts its cytotoxic effect in the G2M phase. Since cyclin A is active and detectable from the beginning of the $S$ phase to the beginning of mitosis, it should theoretically label the proportion of cells that are sensitive to chemotherapeutic drugs used in our study. Despite this, no correlation between cyclin A activity and response rate, TTP and OS calculated from the start of chemotherapy was found. A previous study from a randomised trial comparing adjuvant chemotherapy (cyclophosphamide, methotrexate and 5-fluoro-

\section{REFERENCES}

Anonymous (1998) Polychemotherapy for early breast cancer: an overview of randomized trials. Early Breast Cancer Collaborative Group. Lancet 352: $930-942$

Boiesen P, Bendahl PO, Anagnostaki L, Domanski H, Holm E, Idvall I, Johansson S, Ljungberg O, Ringberg A, Ostberg G, Ferno M (2000) Histologic grading in breast cancer - reproducibility between seven pathologic departments. South Sweden Breast Cancer Group. Acta Oncol 39(1): $41-45$

Bukholm I, Bukholm G, Nesland JM (2001) Over-expression of cyclin A is highly associated with early relapse and reduced survival in patients with primary breast carcinomas. Int J Cancer 93: 283-287

Crown J, Dieras V, Kaufmann M, von Minckwitz G, Kaye S, Leonard R, Marty M, Misset J, Osterwalder B, Piccart M (2002) Chemotherapy for metastatic breast cancer - report of a European expert panel. Lancet Oncol 3(12): 719-727

Elston CW, Ellis IO (1991) Pathological prognostic factors in breast cancer. I. The value of the histological grade in breast cancer: experience from a large study with long-term follow up. Histopathology 19: $403-410$

Fan J, Bertino J (1997) Functional roles of E2F in cell cycle regulation. Oncogene 14: $1191-1200$

Genestie C, Zafrani B, Asselain B, Fourquet A, Rozan S, Validire P, Vincent-Salomon A, Sastre-Garau X (1998) Comparison of the prognostic value of Scarff Bloom Richardsson and Nottingham histological grades in a series of 825 cases of breast cancer: major importance of the mitotic count as a component of both grading systems. Anticancer Res 18: $571-576$

Hahka-Kemppinen M, Muhonen T, Nordling S, Pyrhönen S (1997) DNA flow cytometry and the outcome of chemoimmunotherapy in metastatic melanoma. Melanoma Res 7: 329-334

Huuhtanen R, Wiklund T, Blomqvist C, Böhling T, Virolainen M, Tribukait $\mathrm{B}$, Andersson LC (1999) A high proliferation rate measured by cyclin A predicts a favourable chemotherapy response in soft tissue sarcoma patients. Br J Cancer 6: $1017-1021$

Joensuu H, Ristamäki R, Söderström K, Jalkanen S (1994) Effect of treatment on the prognostic value of S-phase reaction in non-Hodgkin lymphoma. J Clin Oncol 12: 2167-2175 uracil) to radiotherapy in primary breast cancer showed that the benefit from chemotherapy was more pronounced among patients with tumours with a high S-phase fraction (Stal et al, 1994). Thus, the strong contrast between the adverse prognostic impact of a high proliferation rate from diagnosis of the disease, but not after start of aggressive chemotherapy, may indicate that chemotherapy has improved the course of the disease, especially in highly proliferating tumours.

We conclude that cyclin A seems to be the strongest prognostic factor in a panel of proliferation markers including Ki-67 and mitotic count in metastatic breast cancer. None of the proliferation markers predicted chemotherapy response to the three regimens in the study.

Kuhling H, Alm P, Olsson H, Fernö M, Baldetorp B, Parwarech R, Rudolph P (2003) Expression of cyclins E, A, and B, and prognosis in lymph nodenegative breast cancer. $J$ Pathol 199: $424-431$

Michalides R, van Tinteren H, Balkenende A, Vermorken J, Benraadt J, Huldij J, van Diest P (2002) Cyclin A is a prognostic indicator in early stage breast cancer with and without tamoxifen treatment. $\mathrm{Br} J$ Cancer 86(3): $402-408$

Michels J, Duigou F, Marnay J, Henry-Amar M, Delozier T, Denoux Y, Chasle J (2002) Flow cytometry and quantitative immunohistochemical study of cell cycle regulation proteins in invasive breast carsinoma. Cancer 97: $1376-1386$

Miller AB, Hoogstraten B, Staquet M, Winkler A (1981) Reporting results of cancer treatment. Cancer (Phila) 47: 207-214

Rudolph P, Kuhling H, Alm P, Fernö M, Baldentorp B, Olsson H, Parwaresch R (2003) Differential prognostic impact of the cyclines E and $\mathrm{B}$ in premenopausal and postmenopausal women with lymph-node negative breast cancer. Int J Cancer 105: 674-680

Sjöström J (2002) Predictive factors for response to chemotherapy in advanced breast cancer. Acta Oncol 41: $334-345$

Sjöström J, Blomqvist C, Mouridsen H, Pluzanska A, Ottosson LS, Bengtsson NO, Ostenstad B, Mjaaland I, Palm SM, Wist E, Valvere V, Anderson H, Bergh J (1999) Docetaxel compared with sequential methotrexate and 5fluorouracil in patients with advanced breast cancer after anthracycline failure: a randomised phase III study with crossover on progression by the Scandinavian Breast Group. Eur J Cancer 35: 1194-1201

Stal O, Skoog L, Rutqvist LE, Carstensen JM, Wingren S, Sullivan S, Andersson AC, Dufmats M, Nordenskjold B (1994) S-phase fraction and survival benefit from adjuvant chemotherapy or radiotherapy of breast cancer. Br J Cancer 70(6): 1258-1262

van Diest P, van der Wall E, Baak J (2004) Prognostic value of proliferation in invasive breast cancer: a review. J Clin Pathol 57: 675-681

Volm M, Koomagi R, Mattern J, Stammler G (1997) Cyclin A is associated with an unfavorable outcome in patients with non-small-cell lung carcinomas. $\mathrm{Br} J$ Cancer 75: $1774-1778$

Zindy F, Lamas E, Chenivesse X, Sobczak J, Wang J, Fesquet D, Henglein B, Brechot C (1992) Cyclin A is required in S phase in normal epithelial cells. Biochem Biophys Res Commun 182: 1144-1154 